\title{
Competitiveness of Winter Rice Varieties against Weed under Dry Direct Seeded Conditions
}

\author{
A. N. M. Atikur Rahman ${ }^{1,2}$, A. K. M. Mominul Islam ${ }^{1,2}{ }^{*}$, Md. Altamas Arefin ${ }^{1,2}$, \\ Md. Rashedur Rahman², Md. Parvez Anwar ${ }^{1,2}$ \\ ${ }^{1}$ Weed Management Laboratory, Department of Agronomy, Bangladesh Agricultural University, Mymensingh, Bangladesh \\ ${ }^{2}$ Department of Agronomy, Bangladesh Agricultural University, Mymensingh, Bangladesh \\ Email: *akmmominulislam@bau.edu.bd
}

How to cite this paper: Rahman, A.N.M.A., Islam, A.K.M.M., Arefin, Md.A., Rahman, Md.R. and Anwar, Md.P. (2017) Competitiveness of Winter Rice Varieties against Weed under Dry Direct Seeded Conditions. Agricultural Sciences, 8, 1415-1438. https://doi.org/10.4236/as.2017.812101

Received: November 23, 2017 Accepted: December 26, 2017 Published: December 29, 2017

Copyright (ङ 2017 by authors and Scientific Research Publishing Inc. This work is licensed under the Creative Commons Attribution-NonCommercial International License (CC BY-NC 4.0). http://creativecommons.org/licenses/by-nc/4.0/

\begin{abstract}
Aerobic rice or dry direct seeded rice is highly vulnerable to weeds because of lack of "head start" over weeds and standing water layer to suppress weeds. The risks of chemical control and the huge cost involvement in mechanical control demand an eco-friendly and cost-effective integrated weed management. Weed competitive rice cultivar may be considered as a viable tool for integrated weed management. In these circumstances, an experiment was designed to evaluate weed competitiveness of some selected winter rice varieties under aerobic soil conditions. The study was conducted during dry season (February to June) 2016 at the Agronomy Field Laboratory and Weed Management Laboratory, Bangladesh Agricultural University, Mymensingh. Fourteen rice varieties namely, BRRI dhan28, BRRI dhan29, BRRI dhan47, BRRI dhan50, BRRI dhan55, BRRI dhan58, BRRI dhan59, BRRI dhan67, Binadhan5, Binadhan-6, Binadhan-8, Binadhan-10, BRRI hybrid dhan3 and Agrodhan14 were grown under weedy and weed-free conditions. Plots with no rice were also maintained to observe the natural growth of weed in absence of rice. The experiment was conducted with split-plot design with three replications. Weeding regime was allocated in main plot and rice variety was allocated in sub-plot. Results showed that rice varieties varied widely in their yield performances and weed competitiveness. Among rice varieties, BRRI dhan59 allowed the minimum weed growth $\left(19.2 \mathrm{~g} \mathrm{~m}^{-2}\right)$ while Binadhan-5 allowed the maximum weed growth $\left(62.8 \mathrm{~g} \mathrm{~m}^{-2}\right)$. Grain yield ranged from $1.85 \mathrm{t} \mathrm{ha}^{-1}$ (BRRI dhan55) to $3.92 \mathrm{t} \mathrm{ha}^{-1}$ (Binadhan-5) under weed-free condition and between $0.41 \mathrm{t} \mathrm{ha}^{-1}$ (BRRI dhan55) and $2.06 \mathrm{t} \mathrm{ha}^{-1}$ (BRRI dhan59) under weedy condition. Weed inflicted relative yield loss ranged from $43.4 \%$ to $82.1 \%$ among the varieties. BRRI dhan59 allowed the least yield penalty (43.4\%)
\end{abstract}


while Binadhan-5 had the maximum yield penalty $(82.1 \%)$ due to weed competition. Although Binadhan-5 is the most productive variety $\left(3.92 \mathrm{t} \mathrm{ha}^{-1}\right)$ for aerobic culture but its weed inflicted relative yield loss is higher $(82.1 \%)$ than many other varieties with low yield potential. On the other hand, BRRI dhan59 appeared as the most weed competitive variety (only $43.4 \%$ relative yield loss) with productivity of $3.84 \mathrm{t} \mathrm{ha}^{-1}$. Therefore, weed competitive variety should be considered as a vital tool while designing integrated weed management system for aerobic rice.

\section{Keywords}

Aerobic Rice, Rice-Weed Competition, Dryland Farming, Weed Pressure, Weed Management

\section{Introduction}

Rice (Oryza sativa L.) is the principal food for more than $50 \%$ people and contributes more than $60 \%$ and $25 \%$ to the cereals production of Asia and of the world, respectively, and it formulates nearly $30 \%$ of all the food being consumed in Asia [1]. Globally, rice crop occupies 158 million hectares of the arable land. The global production and productivity of rice is 744.9 million tons and 4.71 ton per hectare, respectively [2]. It is the major food item for billion residents of Asia and is the principal source of nutrition for many of the world's densely populated countries such as China and Bangladesh [3]. In fact, the approximate contribution of rice to the total calories being consumed is $30,30,50,70,60,50$, and $30 \%$ for China, India, Indonesia, Bangladesh, Vietnam, Philippines, and South Korea, respectively [1]. It is reported that, nearly a half of the world population consumes rice as their principal food. Alongside this, the labour force required to produce rice provides livelihood especially to those belonging to the underprivileged. The steady rise in population further underlines the importance of rice. World's rice demand is projected to increase by 50\% from 1997 to 2050 to keep pace with population growth [4].

Compared to other field crops, rice is most widely grown under irrigated condition which accounts for about $50 \%$ of the total amount of water diverted for irrigation, which in itself accounts for $80 \%$ of the amount of fresh water diverted [5]. This is due to the high unproductive water losses by evaporation, surface run-off, and percolation. Producing one kilogram of unprocessed rice grain under irrigation is estimated to use between 1500 and $5000 \mathrm{~L}$ of water, depending on the local climate, soil type and rice variety [6]. This amount is about twice or even more than wheat or maize [7] [8]. However, declining water availability threatens the sustainability of traditional flood-irrigated rice ecosystems [9]. In Asia, it is predicted that 17 million hectares of irrigated rice areas may have "physical water scarcity" and 22 million hectares areas may be subject to "economic water scarcity" by 2025 [10]. In Asia, where about $60 \%$ of the world's 
population lives, food security is challenged by increasing food demand and threatened by declining water availability [7]. It is, therefore, no longer feasible to flood rice fields for better crop establishment and weed control [11]. Among different water-saving approaches, aerobic rice cultivation has come up with a huge success in different parts of the world.

Growing rice in non-saturated and non-puddled aerobic soil is a promising water-wise technique of rice cultivation under the context of ever-mounting water scarcity [3] [12]. Growing rice under aerobic conditions requires 36\% - 41\% less water than under the conventional method [13]. In response to the labour and water shortage problem, some alternative rice production methods were suggested by researchers worldwide including alternate wetting and drying [14] [15], system of rice intensification [16] [17] [18] [19], and raised bed for saturated soil culture [20] [21]. Compared with flooded rice, aerobic rice had lower production cost, higher water productivity, and a comparable outcome [22] [23].

Weeds are a major yield limiting factor in rice production [24], and the literature reporting yield losses is numerous. Globally, actual rice yield losses due to pests have been estimated at $40 \%$, of which weeds have the highest loss potential (32\%). The worldwide estimated rice yield loss due to weeds is around $10 \%$ of the total production [25]. Yield reduction due to weeds is more critical in direct seeded rice than in transplanted rice [26] [27]. In dry seeded aerobic rice, relative yield loss caused by weeds is $50 \%-91 \%$ [28], while in transplanted rice, yield loss has been estimated to be only $13 \%$ [29]. Among the rice ecosystems, yield losses are the highest in aerobic rice [30]. In extreme cases, weed infestation may cause complete failure of aerobic rice [31]. Weeds persist by adapting to cultural practices, and every cultural practice influences the competitive ability of both the crop and weed resulting complex interactions [32]. Cultural approaches play significant role to determine the competitiveness of a crop with weeds for above ground and below ground resources and hence might influence weed management [3] [33] [34] [35] [36]. Integrated weed management strategies offer several options, but risks of developing resistant weed biotypes [37], and environmental hazard resulting from herbicides from chemical control [38] [39] [40] [41], and labor-intensive manual weeding methods [42] demand an eco-friendly and less labor-dependent weed management system for sustainable aerobic rice production.

As observed by many researchers, the performance of herbicides can be enhanced if crop varieties with higher weed competitiveness are used especially in herbicide-dominant systems [27]. Weed competitiveness comprises two components: weed suppressive ability-the ability to lessen weed growth through competition, and weed tolerance-the capability of maintaining potential yields in the presence of weeds [43]. The potentiality of using weed competitive variety in integrated weed management has been documented in many crops including rice [44]-[49]. The deployment of weed competitive variety is not only ecofriendly [50] [51] but also a very cost effective [52] tool for integrated weed 
management. Considering the high vulnerability of aerobic rice to weeds, development of weed competitive aerobic rice variety has been suggested by many researchers [9] [48] [53].

No work has so far been done to assess the ability of the huge pool of Bangladeshi rice germplasms to wrestle weeds under aerobic soil conditions. In this backdrop, the present study was undertaken to study the variation in weed competitiveness among selected high yielding rice varieties and to recognize agronomic traits conferring weed competitiveness of rice grown under aerobic soil conditions.

\section{Materials and Methods}

\subsection{Description of the Experimental Site}

The experiment was carried out at the Agronomy Field Laboratory and Weed Management Laboratory, Department of Agronomy, Bangladesh Agricultural University, Mymensingh during the period from February to June 2016. Geographically the experimental area is located at $24^{\circ} 75^{\prime} \mathrm{N}$ latitude and $90^{\circ} 50^{\prime} \mathrm{E}$ longitudes at the elevation of $18 \mathrm{~m}$ above the sea level. The experimental field was medium high land belonging to the Sonatola Soil Series of Grey Floodplain soil under the agro-ecological zone of Old Brahmaputra Floodplain (AEZ-9). The region occupies the large area of Brahmaputra sediments which were laid down before the river shifted into its present Jamuna Channel about 200 years ago [54] [55]. The soil of the experimental field was more or less neutral in reaction with $\mathrm{pH}$ value 6.8 , low in organic matter content $(1.96 \%)$ and bulk density $(1.35 \mathrm{~g}$ $\left.\mathrm{cm}^{-3}\right)$. The land type was medium high with silty-loam in texture $(20 \%$ sand, $67 \%$ silt and $13 \%$ clay). Soil contained $0.11 \%$ total N, 50.40 ppm available P, 7.36 ppm available $\mathrm{S}, 0.16 \mathrm{me} \%$ exchangeable $\mathrm{K}$.

During the growing season (February-June, 2016), monthly average maximum temperature, minimum temperature and relative humidity were $27.8^{\circ} \mathrm{C}$ $33.6^{\circ} \mathrm{C}, 17.4^{\circ} \mathrm{C}-26.3^{\circ} \mathrm{C}$ and $73.2 \%-84.4 \%$, respectively, while monthly total rainfall and sunshine hours were $0.3-13.0 \mathrm{~mm}$ and $140.1-171.3 \mathrm{~h}$, respectively.

\subsection{Experimental Treatments}

This was a two factorial experiment, where factor A comprised two weeding regime viz., a) weed free and b) weedy condition. On the other hand, factor B consisted of 14 high yielding rice varieties e.g., i) BRRI dhan28, ii) BRRI dhan29, iii) BRRI dhan47, iv) BRRI dhan50, v) BRRIdhan55, vi) BRRI dhan58, vii) BRRI dhan59, viii) BRRI dhan67, ix) Binadhan-5, x) Binadhan-6, xi) Binadhan-8, xii) Binadhan-10, xiii) BRRI hybrid dhan3 and xiv) Agrodhan-14. A brief description of these varieties is given in Table 1.

\subsection{Experimental Design}

The experiment was laid out in split-plot design with three replications. Weeding regime was allocated in main plot and rice variety was allocated in sub-plot. 
Table 1. Brief description of the rice varieties used in the experiment.

\begin{tabular}{cccccc}
\hline Varieties & Released year $\begin{array}{c}\text { Plant height } \\
(\mathrm{cm})\end{array}$ & $\begin{array}{c}\text { Life duration } \\
(\text { days })\end{array}$ & $\begin{array}{c}\text { Average yield } \\
\left(\mathrm{t} \mathrm{ha}^{-1}\right)\end{array}$ & Special features \\
\hline BRRI dhan28 & 1994 & 90 & 140 & 6.00 & Early maturing, less water requiring \\
BRRI dhan29 & 1994 & 95 & 160 & 7.50 & High yield potential \\
BRRI dhan47 & 2007 & 105 & 152 & 6.00 & Salt tolerant \\
BRRI dhan50 & 2008 & 82 & 155 & 6.00 & Premium quality rice, slightly aromatic \\
BRRI dhan55 & 2011 & 100 & 145 & 7.00 & Salt and drought tolerant \\
BRRI dhan58 & 2012 & 100 & 155 & 7.20 & Tolerant to lodging \\
BRRI dhan59 & 2013 & 83 & 153 & 7.10 & Salt tolerant \\
BRRI dhan67 & 2014 & 100 & 143 & 6.00 & Tolerant to lodging \\
Binadhan-5 & 1998 & $110-115$ & $150-155$ & 7.00 & Bears more tillers \\
Binadhan-6 & 1998 & $110-115$ & $160-165$ & 7.50 & Salt tolerant \\
Binadhan-8 & 2010 & - & $130-135$ & 8.00 & Salt tolerant \\
Binadhan-10 & 2012 & - & $127-132$ & 8.00 & Early maturing \\
BRRI hybrid dhan3 & 2009 & 110 & 145 & 9.00 & Tolerant to lodging and seed shattering \\
Agrodhan-14 (Hybrid), & - & $100-110$ & $140-145$ & $8.5-9.00$ & \\
Pioneer 27P31 & & & & &
\end{tabular}

Source: BRRI [56] and BINA [57].

The unit plot size was $2.5 \mathrm{~m} \times 2.0 \mathrm{~m}$. The distance maintained between blocks was $1.0 \mathrm{~m}$ and unit plots were $0.5 \mathrm{~m}$, respectively. Moreover, three plots were left for weed monoculture, where no rice was grown. Thus, a total of 87 unit plots were maintained.

\subsection{Crop Husbandry}

\section{Seed collection}

Seeds of BRRI dhan28, BRRI dhan29, BRRI dhan47, BRRI dhan50, BRRI dhan55, BRRI dhan58, BRRI dhan59, BRRI dhan67 and BRRI hybrid dhan3 were collected from Bangladesh Rice Research Institute (BRRI), seeds of Agrodhan-14 were collected from Petrochem Agro-industries Ltd. and Binadhan-5, Binadhan6. Binadhan- 8 and Binadhan-10 were collected from Bangladesh Institute of Nuclear Agriculture (BINA), Mymensingh.

\section{Land preparation}

The land was first opened with a power tiller and subsequently leveled by laddering. Weeds and stubbles of the previous crop were collected and removed from the field. Before sowing, the field was prepared by plowing and harrowing to obtain a smooth seedbed.

\section{Manure and fertilizer application}

The land was fertilized with cowdung, urea, triple super phosphate, muriate of potash, gypsum and zinc sulphate at 10000, 220, 120, 75, 60 and $10 \mathrm{~kg} \mathrm{ha}^{-1}$ re- 
spectively. The whole amount of cowdung, triple super phosphate, muriate of potash, gypsum and zinc sulphate were applied at the time of final land preparation. Urea was applied in three equal splits at 30,50 and 70 days after sowing (DAS).

\section{Seed sowing and irrigation}

Sowing was done on 3 February 2016. Before sowing seeds were soaked in water for $24 \mathrm{~h}$ and then were kept in a warm place for $48 \mathrm{~h}$ at $25^{\circ} \mathrm{C} \pm 5^{\circ} \mathrm{C}$ temperature for seed incubation. Sprouted seeds were then sown in field following $25 \mathrm{~cm} \times 15 \mathrm{~cm}$ spacing with $5-6$ seeds hill ${ }^{-1}$. To maintain soils at field capacity throughout the growing period three surface irrigations were provided.

\subsection{Data Collection}

A quadrate of size $0.5 \mathrm{~m} \times 0.5 \mathrm{~m}$ was placed randomly in two places of each weedy plots for collecting weed samples. Weed were clipped at ground level, identified and counted by species, and separately oven dried at $70{ }^{\circ} \mathrm{C}$ to constant weight. Weed density (WD) and weed dry weight (WDW) were expressed as no. $\mathrm{m}^{-2}$ and $\mathrm{g} \mathrm{m}^{-2}$, respectively. Relative contribution of different weed groups (broad-leaved, grasses and sedges) to the weed vegetation in terms of relative density (RD) and relative dry weight (RDW) were also calculated. Dominant weed species were identified using the summed dominance ratio (SDR) computed as follows [58]:

$$
\begin{gathered}
\mathrm{RD}(\%)=\frac{\text { Density of a given weed species }}{\text { Total density of weed }} \times 100 \\
\operatorname{RDW}(\%)=\frac{\text { Dry weight of a given weed species }}{\text { Total weed dry weight }} \times 100 \\
S D R=\frac{R D+R D W}{2}
\end{gathered}
$$

In weedy treatments, weed growth was visually rated weed rating (WR) at 9 weeks after sowing (WAS) on a 1 to 9 scale, with 1 for minimum weed growth and 9 for maximum. Days to flowering (DF) and maturity (DM) of the rice varieties were recorded when $50 \%$ plants in a field started to flower and more than $80 \%$ grains turned golden yellow colour, respectively. The early visual vigour (EVV) of the varieties was measured at 3 WAS. The relative chlorophyll content or greenness of rice leaves was measured at 45 DAS (SPAD 45) using a portable SPAD meter. Data was recorded from the youngest fully expanded leaf or flag leaf of 5 plants in each treatment. The rice plant height at different stages i.e. 15, $30,45,60,75$ DAS and harvesting time were measured from ground level to tip of the uppermost leaf/panicle. The average height of five hills was considered as the height to the plant for each plot and expressed in $\mathrm{cm}$.

The crop was harvested at full maturity i.e. when about $80 \%$ of the seeds became golden yellow in color. Five hills (excluding border hills) were randomly selected in each plot and uprooted before harvesting to record data on yield 
contributing characters e.g., effective tillers hill ${ }^{-1}$, grains panicle ${ }^{-1}$ and 1000-grain weight. The harvested crop of each plot was separately bundled, properly tagged and then brought to the threshing floor. The crop was threshed by pedal thresher. Grains were sun dried and cleaned. Straws were also sun dried properly. Finally, grain and straw yields were converted to ton per hectare. The biological yield $\left(\mathrm{t} \mathrm{ha}^{-1}\right)$, harvest index (\%) and relative yield loss (RYL) (\%) were then calculated from the grain and straw yield using the following equation:

$$
\begin{gathered}
\text { Biological yield }=\text { Grain yield }+ \text { Straw yield } \\
\text { Harvest index }(\%)=\frac{\text { Grain yield }}{\text { Biological yield }} \times 100 \\
\text { RYL }(\%)=\frac{\text { Weed free yield }- \text { Treatment yield }}{\text { Weed free yield }} \times 100
\end{gathered}
$$

\subsection{Statistical Analysis}

The collected data were compiled and tabulated in proper form and were subjected to statistical analysis. Data were analyzed using the analysis of variance (ANOVA) technique with the help of computer package program MSTAT-C and mean differences were adjudged by Duncan's Multiple Range Test [59].

\section{Results}

\subsection{Floristic Composition of Weeds}

Twenty-four weed species belonging to thirteen different families were observed in weedy plots, among which fifteen were broadleaves, seven grasses and two sedges (Table 2). Based on summed dominance ratio (SDR), the five most dominant weed species encountered were Panicum disticum, Alternanthera sessilis, Spilanthes acmella, Paspalum commersonii and Echinochloa crusgalli. Broadleaf weeds contributed $63 \%$ of the total dry matter and $44 \%$ of total density compared to grasses ( $34 \%$ and $54 \%$, respectively) and sedges ( $3 \%$ and $2 \%$, respectively) (Figure 1 and Figure 2).

\subsection{Weed Pressure and Density}

Visual weed rating, weed dry matter and weed density varied significantly among rice varieties (Figure 3 and Figure 4). Maximum weed growth was observed in weed monoculture. In terms of weed rating BRRI dhan59, BRRI dhan67 and Binadhan-10 appeared as the most weeds suppressive since weed ratings against these variety were low $(<4)$ (Table 3 ). Weed growth was rated between 4 and 5 for BRRI dhan50, BRRI dhan58, BRRI hybrid dhan3, Binadhan6 and Agrodhan-14, and between 5 and 6 for BRRI dhan28, BRRI dhan29 and BRRI dhan 47 indicating moderate weed suppressive (Table 3). Weed rating (6 7) in BRRI dhan 55 and Binadhan- 8 and highest weed rating $>7$ in Binadhan-5 signify poor competitiveness against weeds. Weed dry matter followed almost similar trend as visual weed rating. Mean weed pressure across variety was 37.83 
$\mathrm{g} \mathrm{m}^{-2}$ against $92.32 \mathrm{~g} \mathrm{~m}^{-2}$ recorded in weed monoculture, which denotes that on average, rice variety reduced weed pressure by about 59\%. BRRI dhan59 emerged as the most weed suppressive variety reducing weed pressure by $79 \%$ followed by BRRI dhan67 (77\%) and Binadhan-10 (75\%) (Figure 3 and Figure 4). Highest weed pressure of $62.8 \mathrm{~g} \mathrm{~m}^{-2}$ was found in Binadhan-5 which was $32 \%$ less than in weed monoculture. Other varieties were intermediate in suppressing weeds within the range of 41 to $79 \%$. Maximum weed density of $197 \mathrm{~m}^{-2}$ was recorded in weed monoculture. The rice variety did not significantly differ with respect to weed density, which was within a narrow range from 69 to $114 \mathrm{~m}^{-2}$ (Figure 4). The average weed density across variety was $94 \mathrm{~m}^{-2}$ which was $52 \%$ less compared to the weed monoculture.

Table 2. Dominant weed species with family name, type, relative density (RD), relative dry weight (RDW) and summed dominance ratio (SDR) (averaged over all weedy plots).

\begin{tabular}{|c|c|c|c|c|c|c|}
\hline Common name & Scientific name & Family name & Weed type & $\mathrm{RD}(\%)$ & RDW (\%) & SDR (\%) \\
\hline Angta & Panicum disticum & Gramineae & Grass & 20.97 & 8.25 & 14.61 \\
\hline Chanchi & Alternanthera sessilis & Amaranthaceae & Broad leaf & 15.56 & 12.13 & 13.84 \\
\hline Halud nakful & Spilanthes acmella & Compositeae & Broad leaf & 9.47 & 4.59 & 8.03 \\
\hline Gaicha & Paspalum commersonii & Gramineae & Grass & 8.79 & 6.93 & 7.86 \\
\hline Shama & Echinochloa crusgalli & Gramineae & Grass & 4.06 & 12.75 & 7.40 \\
\hline Durba & Cynodon dactylon & Gramineae & Grass & 8.12 & 4.32 & 6.22 \\
\hline Foska begun & Physalis minima & Solanaceae & Broad leaf & 2.03 & 10.05 & 5.35 \\
\hline Lazzabati & Mimosa pudica & Leguminaceae & Broad leaf & 1.32 & 7.63 & 4.48 \\
\hline Anguli ghas & Digitaria sanguinalis & Gramineae & Grass & 6.76 & 1.56 & 4.16 \\
\hline Tit begun & Solanum torvum & Solanaceae & Broad leaf & 4.38 & 4.59 & 3.99 \\
\hline Khude shama & Echinochloa colonum & Gramineae & Grass & 4.73 & 4.77 & 3.75 \\
\hline Pani marich & Polygonum orientale & Polygonaceae & Broad leaf & 3.38 & 3.24 & 3.31 \\
\hline Bon pat & Melochia corchorifolia & Malvaceae & Broad leaf & 1.67 & 5.55 & 3.11 \\
\hline Hazar dana & Phyllanthus niruri & Euphorbiaceae & Broad leaf & 2.03 & 1.21 & 2.62 \\
\hline Biskatali & Polygonum hydropiper & Polygonaceae & Broad leaf & 0.67 & 3.82 & 2.24 \\
\hline Bon tamak & Nicotiana plumbaginifolia & Solanaceae & Broad leaf & 1.04 & 2.19 & 2.19 \\
\hline Bon tula & Sanchu arvensis & Asteraceae & Broad leaf & 0.67 & 1.51 & 1.59 \\
\hline Keshuti & Eclipta alba & Compositae & Broad leaf & 2.03 & 0.69 & 1.36 \\
\hline Bathua & Chenopodium album & Chenopodiaceae & Broad leaf & 0.67 & 1.04 & 0.85 \\
\hline Shial leja & Dysophylla crassicaulis & Lamiaceae & Broad leaf & 0.67 & 0.95 & 0.81 \\
\hline Kanai bashi & Commelina bengalensis & Commelinaceae & Broad leaf & 0.67 & 0.61 & 0.64 \\
\hline Mutha & Cyperus rotundus & Cyperaceae & Sedge & 0.67 & 1.10 & 0.64 \\
\hline Guccha mutha & Cyperus nemoralis & Cyperaceae & Sedge & 0.67 & 0.26 & 0.46 \\
\hline Arail & Leersia hexandra & Gramineae & Grass & 0.67 & 0.26 & 0.46 \\
\hline
\end{tabular}

Here, $\mathrm{RD}=$ Relative density, $\mathrm{RDW}=$ Relative dry weight, $\mathrm{SDR}=$ Summed dominance ratio 
$\square$ Grass $\quad \square$ Sedge $\quad \square$ Broadleaf

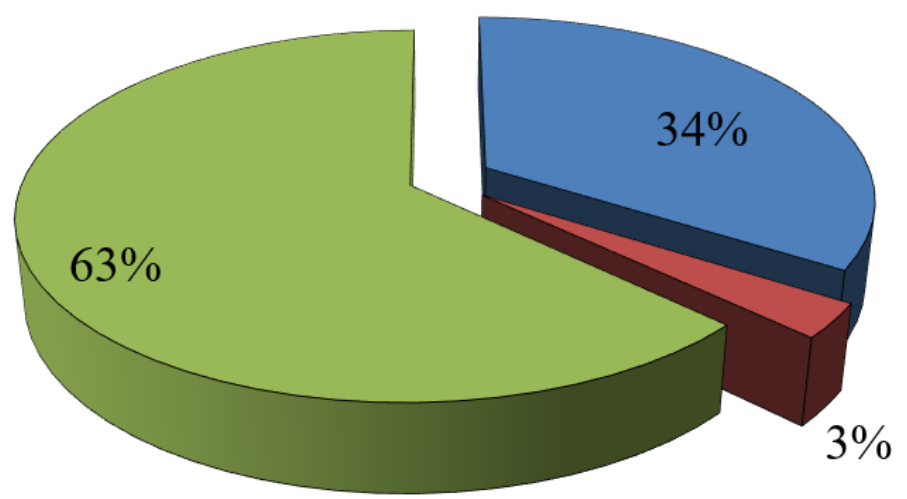

Figure 1. Relative dry weight of different weed groups.

\section{$\square$ Grass $\square$ Sedge $\square$ Broadleaf}
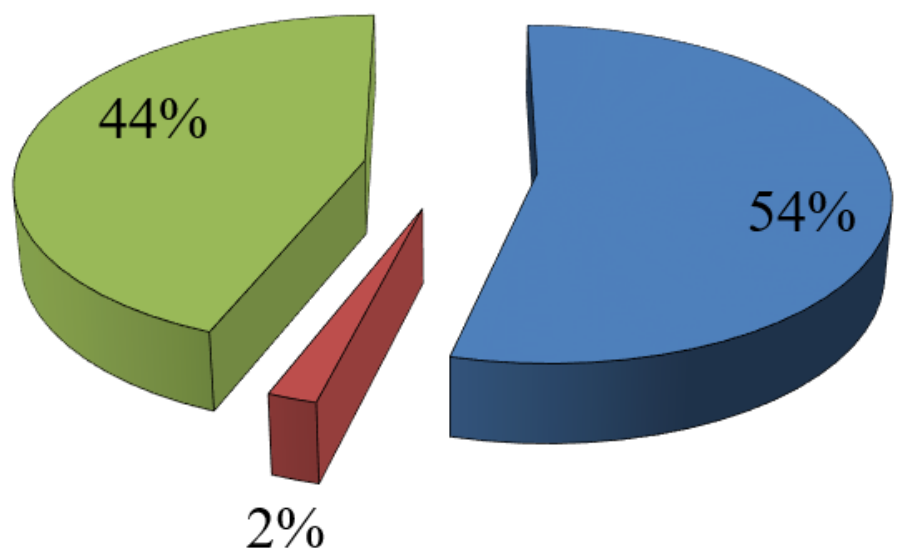

Figure 2. Relative density of different weed groups.

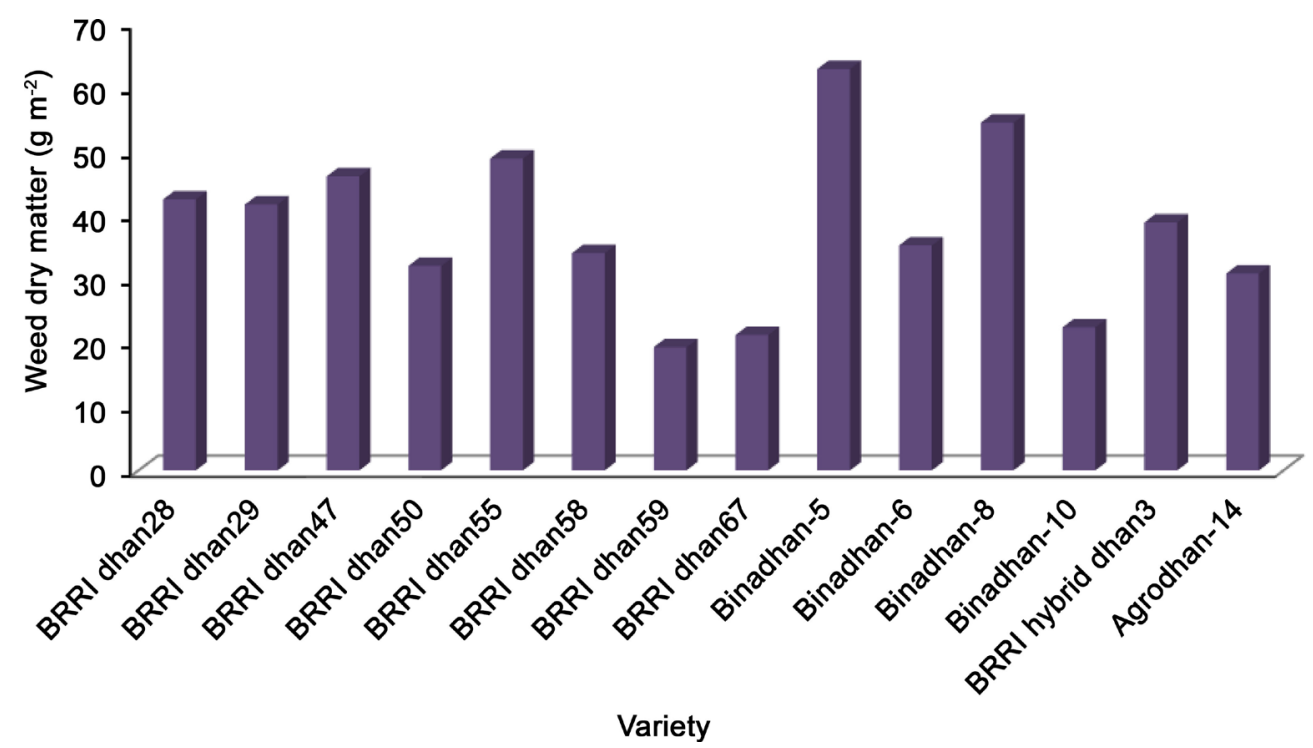

Figure 3. Effect of variety on weed dry weight $\left(\mathrm{g} \mathrm{m}^{-2}\right)$ of winter rice under aerobic system of cultivation. 


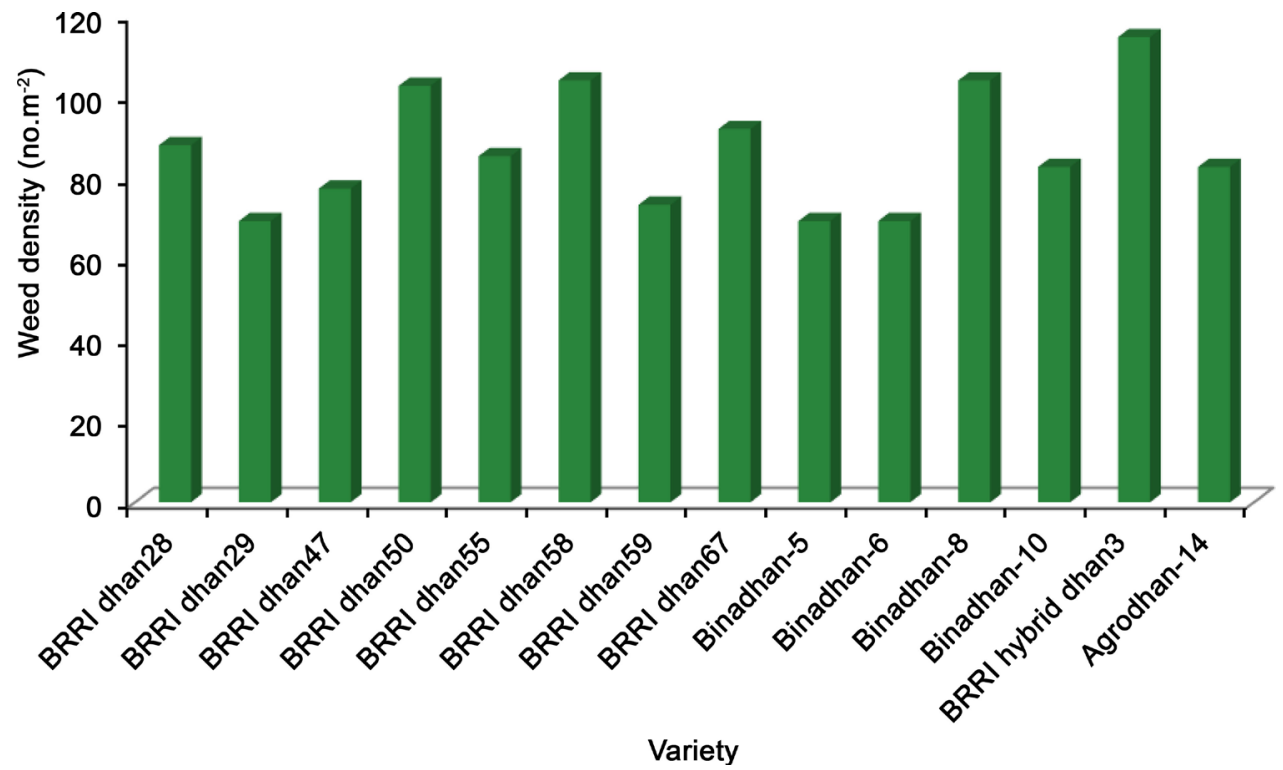

Figure 4. Effect of variety on weed density of winter rice under aerobic system of cultivation.

Table 3. Effect of variety and weeding regime on relative chlorophyll content, early visual vigor and weed rating of winter rice under aerobic system of cultivation.

\begin{tabular}{|c|c|c|c|}
\hline Variety & Chlorophyll content (SPAD value) & EVV at 3 WAS & Weed rating at 9 WAS \\
\hline BRRI dhan 28 & 37.87 & $6.00 \mathrm{de}$ & $5.00 \mathrm{de}$ \\
\hline BRRI dhan29 & 34.46 & 6.00de & $5.00 \mathrm{de}$ \\
\hline BRRI dhan 47 & 35.11 & $5.33 \mathrm{ef}$ & $5.33 \mathrm{~d}$ \\
\hline BRRI dhan50 & 34.15 & 7.17abc & $4.00 \mathrm{~g}$ \\
\hline BRRI dhan55 & 38.50 & $4.67 f$ & $6.00 \mathrm{c}$ \\
\hline BRRI dhan58 & 35.61 & $7.00 \mathrm{a}-\mathrm{d}$ & $4.33 \mathrm{fg}$ \\
\hline BRRI dhan59 & 35.90 & $8.00 \mathrm{a}$ & $3.33 \mathrm{~h}$ \\
\hline BRRI dhan 67 & 32.21 & $7.83 \mathrm{ab}$ & $3.33 \mathrm{~h}$ \\
\hline Binadhan-5 & 35.96 & $3.50 \mathrm{~g}$ & $7.33 \mathrm{a}$ \\
\hline Binadhan-6 & 34.63 & $6.83 \mathrm{bcd}$ & $4.33 \mathrm{fg}$ \\
\hline Binadhan-8 & 32.68 & $4.33 \mathrm{fg}$ & $6.67 b$ \\
\hline Binadhan-10 & 36.13 & $7.50 \mathrm{ab}$ & $3.33 \mathrm{~h}$ \\
\hline BRRI hybrid dhan 3 & 34.48 & $6.17 \mathrm{cde}$ & $4.67 \mathrm{~b}$ \\
\hline Agrodhan-14 & 33.71 & $7.17 \mathrm{abc}$ & $4.00 \mathrm{~g}$ \\
\hline CV\% & 9.67 & 8.48 & 14.21 \\
\hline Level of significance & NS & $* *$ & $* *$ \\
\hline \multicolumn{4}{|l|}{ Weeding Regime } \\
\hline Weed free & $39.22 \mathrm{a}$ & $6.50 \mathrm{a}$ & - \\
\hline Weedy & $30.98 \mathrm{~b}$ & $6.00 \mathrm{~b}$ & - \\
\hline $\mathrm{CV} \%$ & 1.05 & 5.24 & - \\
\hline Level of significance & ** & * & - \\
\hline
\end{tabular}

In a column figures with same letter or without letter do not differ significantly whereas figures with dissimilar letter differ significantly (as per DMRT). ${ }^{*}=$ Significant at $5 \%$ level of probability, ${ }^{* *}=$ Significant at $1 \%$ level of probability, NS $=$ Not significant, WAS $=$ Weeks after sowing. 


\subsection{Relative Chlorophyll Content (SPAD Value)}

Relative chlorophyll content was not significantly influenced by variety (Table 3). Numerically, the highest chlorophyll content was observed in BRRI dhan55 (38.5) and the lowest chlorophyll content was observed in BRRI dhan67 (32.22). Higher SPAD values indicate greener and healthier plants. Relative chlorophyll content was significantly affected by weeding regimes (Table 3 ). In weed free condition higher chlorophyll value was found (39.22) and in weedy treatment lower chlorophyll content (30.98) was found. Weed competition was severe in weedy condition and thus lowest chlorophyll was produced. On the other hand, in weed free treatment throughout the crop growth period, higher chlorophyll value was produced (Table 3). SPAD value was higher in weed-free condition than in weedy condition. SPAD values were greatly reduced by weed interference and this was reflected in yield performance.

\subsection{Early Visual Vigor}

Early visual vigor was significantly influenced by variety (Table 3 ). In the present study, early vigor varied widely among varieties. Early visual vigor ranged from 3.5 to 8.0. The highest early visual vigor was observed in BRRI dhan59 (8.0) and the lowest was observed in Binadhan-5 (3.5). Early visual vigor was significantly affected by weeding regimes (Table 3 ). Higher early visual vigor (6.5) was found in weed free treatment and lower in weedy treatment (6.0).

\subsection{Rice Phenology}

The varieties from diverse genetic sources and origins demonstrated a broad range in phonological parameters (Table 4). Growth duration of the varieties in this study ranged from 117 to 143 days. Average flowering period was 109 days in weed free and 107 days in weedy condition. Average growth duration was 134 and 132 days in weed free and weedy condition, respectively. BRRI dhan28 and BRRI dhan 55 took less than 100 days for flowering and near about 120 days for maturing in both conditions. BRRI dhan67, BRRI dhan 47 and BRRI dhan58 commenced flowering between 100 and 105 DAS and consequently matured by 125-130 days in weed free condition and matured 2-3 days early in weedy condition. BRRI dhan 29 required the longest duration of more than 115 days to initiate flowering and matured after 140 days.

\subsection{Plant Height}

The variety and weeding regime exhibited significant differences in plant height at most of the sampling dates, however, their interaction had no significant effect (Table 5 and Table 6). At 15 DAS plant height ranged from 7.65 to 11.55 $\mathrm{cm}$. Here, highest plant height at 15 DAS was produced by BRRI dhan59, followed by Binadhan-10 and the lowest plant height was obtained from Binadhan5 followed by Binadhan-8. At 30 DAS, plant height ranged from 13.05 to $18.55 \mathrm{~cm}$. 
Table 4. Means for varieties over weeding regimes and for weeding regimes over varieties for days required to flowering and maturity of rice.

\begin{tabular}{ccccc}
\hline \multirow{2}{*}{ Variety } & \multicolumn{2}{c}{ Days to 50\% flowering } & \multicolumn{2}{c}{ Days to maturity } \\
\cline { 2 - 4 } & Weed free & Weedy & Weed free & Weedy \\
\hline BRRI dhan28 & 92 & 90 & 117 & 115 \\
BRRI dhan29 & 118 & 115 & 130 & 140 \\
BRRI dhan47 & 105 & 111 & 138 & 136 \\
BRRI dhan50 & 112 & 95 & 122 & 120 \\
BRRI dhan55 & 97 & 103 & 130 & 128 \\
BRRI dhan58 & 105 & 110 & 138 & 136 \\
BRRI dhan59 & 113 & 98 & 126 & 125 \\
BRRI dhan67 & 100 & 113 & 141 & 138 \\
Binadhan-5 & 115 & 114 & 141 & 139 \\
Binadhan-6 & 116 & 110 & 138 & 135 \\
Binadhan-8 & 113 & 110 & 138 & 136 \\
Binadhan-10 & 112 & 113 & 140 & 137 \\
BRRI hybrid dhan3 & 115 & 115 & 141 & 138 \\
Agrodhan-14 & 116 & 107 & 134 & 132 \\
Average & 109 & & & \\
\hline
\end{tabular}

Table 5. Effect of variety and weeding regime on plant height of winter rice under aerobic system of cultivation.

\begin{tabular}{|c|c|c|c|c|c|c|}
\hline \multirow{2}{*}{ Variety } & \multicolumn{6}{|c|}{ Plant height $(\mathrm{cm})$} \\
\hline & 15 DAS & 30 DAS & 45 DAS & 60 DAS & 75 DAS & Harvest \\
\hline BRRI dhan28 & $8.85 \mathrm{efg}$ & $15.77 \mathrm{bc}$ & 30.25 & $56.87 \mathrm{ab}$ & $63.53 c$ & 80.93def \\
\hline BRRI dhan29 & $9.15 \mathrm{def}$ & $16.60 \mathrm{~b}$ & 33.00 & $62.40 \mathrm{a}$ & $66.13 \mathrm{abc}$ & $82.00 \mathrm{cde}$ \\
\hline BRRI dhan47 & $8.60 \mathrm{efg}$ & $13.25 \mathrm{~d}$ & 31.35 & $61.47 \mathrm{a}$ & $67.58 \mathrm{abc}$ & $82.67 \mathrm{c}$ \\
\hline BRRI dhan 50 & $10.65 a-d$ & $16.60 \mathrm{~b}$ & 29.93 & $58.00 \mathrm{ab}$ & $61.57 \mathrm{c}$ & $74.40 \mathrm{f}$ \\
\hline BRRI dhan55 & $7.65 f g h$ & $13.25 \mathrm{~d}$ & 32.52 & $63.60 \mathrm{a}$ & $68.60 \mathrm{abc}$ & $81.77 \mathrm{c}$ \\
\hline BRRI dhan58 & $9.05 \mathrm{~d}-\mathrm{g}$ & $17.45 \mathrm{ab}$ & 30.82 & $60.80 \mathrm{a}$ & $64.60 \mathrm{bc}$ & $77.50 \mathrm{f}$ \\
\hline BRRI dhan59 & $11.55 \mathrm{a}$ & $18.55 \mathrm{a}$ & 31.52 & $57.52 \mathrm{ab}$ & $65.00 \mathrm{bc}$ & 80.13def \\
\hline BRRI dhan67 & $10.80 \mathrm{abc}$ & $17.70 \mathrm{ab}$ & 31.62 & $64 . .97 a$ & $72.63 \mathrm{ab}$ & $84.03 \mathrm{~b}-\mathrm{e}$ \\
\hline Binadhan-5 & $6.80 \mathrm{~h}$ & $13.05 \mathrm{~d}$ & 32.22 & $60.43 a$ & $70.00 \mathrm{abc}$ & $88.23 \mathrm{abc}$ \\
\hline Binadhan-6 & $9.20 c-f$ & $17.00 \mathrm{ab}$ & 33.15 & $64.00 \mathrm{a}$ & $73.63 \mathrm{ab}$ & $90.27 \mathrm{ab}$ \\
\hline Binadhan-8 & $7.45 \mathrm{gh}$ & $14.55 \mathrm{~cd}$ & 30.15 & $61.30 \mathrm{a}$ & $69.87 \mathrm{abc}$ & $82.97 \mathrm{cde}$ \\
\hline Binadhan-10 & $10.90 \mathrm{ab}$ & $17.10 \mathrm{ab}$ & 32.15 & $58.87 \mathrm{a}$ & $64.83 \mathrm{bc}$ & $79.23 \mathrm{def}$ \\
\hline BRRI hybrid dhan3 & $8.70 \mathrm{efg}$ & $14.05 \mathrm{~cd}$ & 32.52 & $61.93 \mathrm{a}$ & $74.53 \mathrm{a}$ & $84.53 \mathrm{bcd}$ \\
\hline Agrodhan-14 & $9.70 \mathrm{~b}-\mathrm{e}$ & $15.95 b c$ & 32.70 & $62.70 \mathrm{a}$ & $75.10 \mathrm{a}$ & $91.97 \mathrm{a}$ \\
\hline $\mathrm{CV} \%$ & 8.69 & 6.05 & 9.15 & 6.95 & 6.56 & 4.18 \\
\hline Level of significance & $* *$ & $* *$ & NS & * & $* *$ & $* *$ \\
\hline \multicolumn{7}{|l|}{ Weeding Regime } \\
\hline Weed free & 9.35 & $17.24 \mathrm{a}$ & $34.19 \mathrm{a}$ & $61.69 \mathrm{a}$ & $69.06 \mathrm{a}$ & $87.42 \mathrm{a}$ \\
\hline Weedy & 9.09 & $14.32 \mathrm{~b}$ & $29.22 b$ & $59.72 b$ & $66.89 \mathrm{~b}$ & $78.39 \mathrm{~b}$ \\
\hline CV\% & 12.82 & 9.83 & 7.84 & 14.11 & 15.81 & 5.55 \\
\hline Level of significance & NS & * & $*$ & * & * & * \\
\hline
\end{tabular}

Other details are same as Table 3. 
A. N. M. A. Rahman et al.

Table 6. Interaction effect of variety and weeding regime on plant height of winter rice under aerobic system of cultivation.

\begin{tabular}{|c|c|c|c|c|c|c|}
\hline \multirow{2}{*}{ Interaction (Variety $\times$ Weeding regime) } & \multicolumn{6}{|c|}{ Plant height $(\mathrm{cm})$} \\
\hline & 15 DAS & 30 DAS & 45 DAS & 60 DAS & 75 DAS & Harvest \\
\hline BRRI dhan28 & 9.00 & 17.40 & 33.03 & $55.37 \mathrm{~b}$ & 62.33 & 86.73 \\
\hline BRRI dhan29 & 9.20 & 18.30 & 34.96 & $65.73 \mathrm{ab}$ & 66.33 & 87.53 \\
\hline BRRI dhan 47 & 8.90 & 14.70 & 34.73 & $63 . .07 \mathrm{ab}$ & 68.33 & 89.26 \\
\hline BRRI dhan 50 & 10.80 & 18.50 & 34.63 & $61.13 \mathrm{ab}$ & 65.13 & 77.13 \\
\hline BRRI dhan55 & 7.70 & 14.50 & 35.20 & $66.13 \mathrm{ab}$ & 71.00 & 85.33 \\
\hline BRRI dhan58 & 9.10 & 18.70 & 31.76 & $58.00 \mathrm{ab}$ & 64.60 & 81.26 \\
\hline BRRI dhan59 & 11.80 & 19.80 & 32.16 & $55.47 \mathrm{~b}$ & 64.20 & 82.13 \\
\hline BRRI dhan67 & 10.90 & 18.90 & 33.90 & $59.67 \mathrm{ab}$ & 74.46 & 89.66 \\
\hline Binadhan-5 & 6.90 & 14.80 & 34.43 & $58.20 \mathrm{ab}$ & 69.93 & 93.20 \\
\hline Binadhan-6 & 9.50 & 18.40 & 37.00 & $69.20 \mathrm{a}$ & 72.20 & 95.20 \\
\hline Binadhan-8 & 7.70 & 16.30 & 32.73 & $62.33 \mathrm{ab}$ & 70.06 & 86.86 \\
\hline Binadhan-10 & 10.90 & 18.30 & 34.73 & $62.93 \mathrm{ab}$ & 67.80 & 85.66 \\
\hline BRRI hybrid dhan3 & 8.70 & 15.40 & 34.83 & $64.53 \mathrm{ab}$ & 73.26 & 86.33 \\
\hline Agrodhan-14 & 9.80 & 17.30 & 34.50 & $68.60 \mathrm{a}$ & 77.13 & 97.53 \\
\hline BRRI dhan 28 & 8.70 & 14.13 & 27.46 & $58.27 \mathrm{ab}$ & 64.73 & 75.13 \\
\hline BRRI dhan29 & 9.10 & 14.90 & 31.03 & $59.07 \mathrm{ab}$ & 65.93 & 76.53 \\
\hline BRRI dhan 47 & 8.30 & 11.80 & 27.96 & $59.87 \mathrm{ab}$ & 66.83 & 76.06 \\
\hline BRRI dhan50 & 10.50 & 14.70 & 25.23 & $54.87 \mathrm{~b}$ & 58.00 & 71.66 \\
\hline BRRI dhan55 & 7.60 & 12.00 & 29.83 & $61.07 \mathrm{ab}$ & 66.20 & 78.20 \\
\hline BRRI dhan58 & 9.00 & 16.20 & 29.86 & $63.60 \mathrm{ab}$ & 64.60 & 73.73 \\
\hline BRRI dhan59 & 11.30 & 17.30 & 30.86 & $59.67 \mathrm{ab}$ & 65.80 & 78.13 \\
\hline BRRI dhan67 & 10.70 & 16.50 & 29.33 & 61.33ab & 70.80 & 78.40 \\
\hline Binadhan-5 & 6.70 & 11.30 & 30.00 & $62.67 \mathrm{ab}$ & 70.06 & 83.26 \\
\hline Binadhan-6 & 8.90 & 15.60 & 29.30 & $58.80 \mathrm{ab}$ & 70.13 & 85.333 \\
\hline Binadhan-8 & 7.20 & 12.80 & 27.56 & $60.27 \mathrm{ab}$ & 69.66 & 79.067 \\
\hline Binadhan-10 & 10.90 & 15.90 & 29.56 & $54.80 \mathrm{~b}$ & 61.86 & 72.8 \\
\hline BRRI hybrid dhan 3 & 8.70 & 12.70 & 30.20 & $59.33 \mathrm{ab}$ & 66.00 & 82.73 \\
\hline Agrodhan-14 & 9.60 & 14.60 & 30.90 & $62.47 \mathrm{ab}$ & 75.80 & 86.40 \\
\hline CV\% & 8.69 & 6.05 & 9.15 & 6.95 & 6.56 & 4.18 \\
\hline Level of significance & NS & NS & NS & ** & NS & NS \\
\hline
\end{tabular}

Other details are same as Table 3.

Here, BRRI dhan 59 was the tallest variety and Binadnan- 5 was the shortest one which was at par with BRRI dhan47, BRRI dhan55, Binadhan-8 and BRRI hybrid dhan3. At 45 DAS, values are not significant. Both at 60 and 75 DAS, Agrodhan-14 was the tallest cultivar which was followed by BRRI hybrid dhan3, and 
BRRI dhan28 was the shortest one which was at par with BRRI dhan59 and BRRI dhan50. At harvest, plant height ranged from 74.4 to $91.97 \mathrm{~cm}$. Agrodhan14 appeared as the tallest variety which was at par with Binadhan-6, and BRRI dhan 50 was the shortest variety which was closely followed by BRRI dhan 58 . Weed infestation reduced plant height at all growth stages. The magnitude of reduction varied with growth phase, and reduction in plant height in weedy treatments followed a declining trend with advancement of crop growth. Presence of weeds markedly decreased plant height by $3,16,14,3.5,3.5$ and $10 \%$ at $15,30,45,60,75$ DAS and harvesting time, respectively. In case of interaction, the tallest variety was Binadhan-6 $(69.2 \mathrm{~cm})$ which was followed by Agrodhan-14 $(68.6 \mathrm{~cm})$ in weed free condition at 60 DAS. The shortest variety was Binadhan-10 $(54.8 \mathrm{~cm})$ which was statistically similar with BRRI dhan50 in weedy condition and BRRI dhan28 and BRRI dhan59 in weed free condition (Table 6).

\subsection{Yield Contributing Characters and Yield}

Except 1000-grain weight, all other yield contributing characters and yield were significantly influenced by variety, weeding regime and their interactions (Table 7 and Table 8). The highest number of effective tillers hill ${ }^{-1}$ and 1000-grain weight was observed in BRRI dhan28 (7.95) and Binadhan-5 (25.55 g), respectively. However, both of this parameter was statistically identical with BRRI dhan59 (7.77 and 24.7 g, respectively). The highest grains panicle ${ }^{-1}$ (55), grain yield $\left(2.95 \mathrm{t} \mathrm{ha}^{-1}\right)$ and biological yield $\left(6.80 \mathrm{t} \mathrm{ha}^{-1}\right)$ was found in BRRI dhan 59 (Table 7). The lowest number of number of grains panicle ${ }^{-1}$ (24.9), grain yield $\left(1.10 \mathrm{t} \mathrm{ha}^{-1}\right)$ and biological yield $\left(2.55 \mathrm{t} \mathrm{ha}^{-1}\right)$ was found in BRRI dhan55. But the lowest number of effective tillers hill ${ }^{-1}$ and 1000-grain weight was observed in Binadhan-8 (4.62) and BRRI dhan29 (19.03 g), respectively. On the other hand, weed free treatment gave the higher values for all the yield contributing characters and yield, and weedy treatment performed the lowest (Table 7). In case of interaction, the highest number of number of effective tillers hill ${ }^{-1}$ (11.67) and grains panicle ${ }^{-1}$ (66.5) were observed in BRRI dhan28 and BRRI dhan29, respectively under weed free treatment (Table 8). In contrast, highest 1000-grain weight, grain and biological yield were observed in Binadhan-5 under the same condition. Moreover, grain yield of BRRI dhan59 and BRRI dhan47 was statistically identical with Binadhan-5 under weed free condition. BRRI dhan55 performed the lowest under weedy treatment for most of the yield contributing characters and yield (Table 8).

\subsection{Harvest Index (\%)}

Variety and weeding regime had significant effect on harvest index however; their interaction had no significant effect on harvest index (Table 7 and Table 8). Harvest index ranged from 40.73 to $42.78 \%$. The highest harvest index was found in BRRI dhan59 (42.78\%) and the lowest one was found in BRRI dhan 28 (40.73\%). Higher harvest index (45.49\%) was found in weed free treatment. 
Table 7. Effect of variety and weeding regime on different yield contributing characters and yield of winter rice under aerobic system of cultivation.

\begin{tabular}{|c|c|c|c|c|c|c|}
\hline Variety & $\begin{array}{l}\text { Effective tillers } \\
\text { hill }^{-1} \text { (no.) }\end{array}$ & $\begin{array}{c}\text { Grains panicle }{ }^{-1} \\
\text { no.) }\end{array}$ & $\begin{array}{l}1000 \text { grain } \\
\text { weight ( } g \text { ) }\end{array}$ & $\begin{array}{l}\text { Grain yield } \\
\qquad\left(\mathrm{t} \mathrm{ha}^{-1}\right)\end{array}$ & $\begin{array}{l}\text { Biological yield } \\
\qquad\left(\mathrm{t} \mathrm{ha}^{-1}\right)\end{array}$ & $\begin{array}{c}\text { Harvest index } \\
(\%)\end{array}$ \\
\hline BRRI dhan28 & $7.95 a$ & $36.90 \mathrm{fg}$ & $19.15 c$ & $2.11 \mathrm{ef}$ & $4.97 \mathrm{def}$ & 42.45 \\
\hline BRRI dhan29 & $6.60 \mathrm{~cd}$ & $54.60 \mathrm{a}$ & $19.03 c$ & $2.28 \mathrm{~cd}$ & $5.19 \mathrm{de}$ & 43.93 \\
\hline BRRI dhan 47 & $5.467 \mathrm{ef}$ & $49.69 \mathrm{~b}$ & $21.85 \mathrm{abc}$ & $2.30 \mathrm{~cd}$ & $5.20 \mathrm{~d}$ & 44.23 \\
\hline BRRI dhan50 & $6.13 \mathrm{de}$ & $48.15 b$ & $20.15 b c$ & $2.16 \mathrm{def}$ & $4.94 \mathrm{ef}$ & 41.53 \\
\hline BRRI dhan55 & $4.967 f$ & $24.90 \mathrm{~h}$ & $20.60 \mathrm{bc}$ & $1.103 \mathrm{i}$ & $2.55 \mathrm{i}$ & 43.13 \\
\hline BRRI dhan58 & $6.33 \mathrm{de}$ & $33.95 \mathrm{~g}$ & $22.42 \mathrm{abc}$ & $1.77 \mathrm{~g}$ & $4.04 \mathrm{~g}$ & 43.81 \\
\hline BRRI dhan59 & $7.76 \mathrm{ab}$ & $55.00 \mathrm{a}$ & $24.70 \mathrm{ab}$ & $2.95 \mathrm{a}$ & $6.80 \mathrm{a}$ & 43.38 \\
\hline BRRI dhan 67 & $7.46 a b c$ & $43.45 \mathrm{~cd}$ & $21.85 \mathrm{abc}$ & $2.62 \mathrm{~b}$ & $6.04 \mathrm{~b}$ & 43.37 \\
\hline Binadhan-5 & $6.46 \mathrm{~d}$ & $47.65 \mathrm{~b}$ & $25.50 \mathrm{a}$ & $2.31 \mathrm{~cd}$ & $5.19 \mathrm{de}$ & 44.51 \\
\hline Binadhan-6 & $7.03 a-d$ & $40.90 \mathrm{de}$ & $22.10 \mathrm{abc}$ & $2.24 \mathrm{de}$ & $5.14 \mathrm{de}$ & 43.15 \\
\hline Binadhan-8 & $4.61 \mathrm{f}$ & $38.65 \mathrm{ef}$ & $20.20 \mathrm{bc}$ & $1.34 \mathrm{~h}$ & $3.03 \mathrm{~h}$ & 44.22 \\
\hline Binadhan-10 & $6.98 \mathrm{bcd}$ & $47.10 \mathrm{bc}$ & $21.10 \mathrm{abc}$ & $2.39 \mathrm{c}$ & $5.49 \mathrm{c}$ & 43.53 \\
\hline BRRI hybrid dhan3 & 6.30de & $39.35 \mathrm{ef}$ & $23.50 \mathrm{abc}$ & $2.23 \mathrm{de}$ & $5.05 \mathrm{de}$ & 44.15 \\
\hline Agrodhan-14 & $6.51 \mathrm{~cd}$ & $36.40 \mathrm{fg}$ & $22.30 \mathrm{abc}$ & $2.07 f$ & $4.74 \mathrm{f}$ & 43.67 \\
\hline $\mathrm{CV} \%$ & 12.75 & 8.02 & 10.42 & 5.88 & 4.62 & 5.17 \\
\hline Level of significance & $* *$ & ** & $* *$ & ** & $* *$ & NS \\
\hline \multicolumn{7}{|l|}{ Weeding Regime } \\
\hline Weed Free & $8.43 a$ & $49.40 \mathrm{a}$ & 23.63 & $3.18 \mathrm{a}$ & $6.997 a$ & $45.49 \mathrm{a}$ \\
\hline Weedy & $4.52 \mathrm{~b}$ & $35.84 \mathrm{~b}$ & 19.87 & $1.10 \mathrm{~b}$ & $2.77 \mathrm{~b}$ & $39.35 b$ \\
\hline $\mathrm{CV} \%$ & 2.48 & 18.71 & 26.64 & 12.43 & 6.22 & 6.67 \\
\hline Level of significance & ** & * & NS & ** & ** & ** \\
\hline
\end{tabular}

Other details are same as Table 3.

Lower harvest index (39.35\%) was found in weedy treatment (Table 7). Harvest index percentage was ranged from 35.99 to 45.53 . Numerically, highest harvest index was produced by BRRI dhan59 (45.53\%) in weed free treatment, and the lowest one (35.99\%) was found in BRRI dhan28 under weedy condition (Table 8).

\subsection{Relative Yield Loss}

Relative yield loss is an excellent indicator of weed tolerance of a variety. Lower the relative yield loss, higher the degree of weed tolerance, since weed tolerance refers to the ability to maintain high yield in the presence of weed competition. The rice varieties showed wide diversity in relative yield loss, which ranged from $43.4 \%$ to $82.1 \%$ (Figure 5). The relative yield loss was lowest in BRRI dhan59, followed by BRRI dhan67 and Binadhan-10 which exhibited high weed tolerance. 
Binadhan-5 had the lowest tolerance to weeds with a yield penalty of $82.1 \%$ closely followed by Binadhan-8, BRRI dhan55 and others (Figure 5).

Table 8. Interaction effect of variety and weeding regime on different yield contributing characters and yield of winter rice under aerobic system of cultivation.

\begin{tabular}{|c|c|c|c|c|c|c|}
\hline $\begin{array}{c}\text { Interaction } \\
(\text { Variety } \times \text { Weeding regime })\end{array}$ & $\begin{array}{l}\text { Effective tillers } \\
\text { hill }^{-1} \text { (no.) }\end{array}$ & $\begin{array}{l}\text { Grains panicle }{ }^{-1} \\
\text { (no.) }\end{array}$ & $\begin{array}{l}1000 \text { grain } \\
\text { weight }(g)\end{array}$ & $\begin{array}{l}\text { Grain yield } \\
\left(\mathrm{t} \mathrm{ha}^{-1}\right)\end{array}$ & $\begin{array}{l}\text { Biological yield } \\
\qquad\left(\mathrm{t} \mathrm{ha}^{-1}\right)\end{array}$ & $\begin{array}{l}\text { Harvest index } \\
(\%)\end{array}$ \\
\hline BRRI dhan28 & $11.67 \mathrm{a}$ & $42.30 \mathrm{~h}-\mathrm{k}$ & 20.80 & $3.27 \mathrm{~cd}$ & $7.19 \mathrm{~d}$ & 45.46 \\
\hline BRRI dhan29 & $9.66 \mathrm{~b}$ & $66.50 \mathrm{a}$ & 18.46 & $3.47 \mathrm{bc}$ & $7.63 c$ & 45.51 \\
\hline BRRI dhan47 & 7.86de & $54.90 \mathrm{bcd}$ & 24.30 & $3.73 \mathrm{a}$ & $8.20 \mathrm{~b}$ & 45.51 \\
\hline BRRI dhan 50 & 7.73de & $57.40 \mathrm{bc}$ & 21.50 & $3.18 \mathrm{de}$ & $6.99 \mathrm{~d}$ & 45.48 \\
\hline BRRI dhan55 & $6.86 \mathrm{ef}$ & $28.30 \mathrm{o}$ & 23.90 & $1.85 \mathrm{~h}$ & $4.07 \mathrm{i}$ & 45.44 \\
\hline BRRI dhan58 & $8.46 \mathrm{bcd}$ & $38.70 \mathrm{j}-\mathrm{m}$ & 24.63 & $2.65 f$ & $5.83 \mathrm{f}$ & 45.47 \\
\hline BRRI dhan59 & $8.76 \mathrm{bcd}$ & $57.20 \mathrm{bc}$ & 26.60 & $3.84 \mathrm{a}$ & $8.44 \mathrm{ab}$ & 45.53 \\
\hline BRRI dhan67 & $8.73 \mathrm{bcd}$ & 50.50def & 23.40 & $3.47 \mathrm{bc}$ & $7.63 c$ & 45.48 \\
\hline Binadhan-5 & $9.53 b c$ & $58.60 \mathrm{~b}$ & 27.70 & $3.92 \mathrm{a}$ & $8.62 \mathrm{a}$ & 45.50 \\
\hline Binadhan-6 & $8.26 \mathrm{~cd}$ & 50.50def & 23.70 & $3.18 \mathrm{de}$ & $7.03 \mathrm{~d}$ & 45.44 \\
\hline Binadhan-8 & $6.26 f g$ & $48.40 \mathrm{efg}$ & 23.50 & $2.22 \mathrm{~g}$ & $4.88 \mathrm{~g}$ & 45.51 \\
\hline Binadhan-10 & 7.76de & $52.80 \mathrm{cde}$ & 22.60 & $3.23 \mathrm{~d}$ & $7.10 \mathrm{~d}$ & 45.50 \\
\hline BRRI hybrid dhan 3 & $8.33 \mathrm{bcd}$ & $45.20 \mathrm{fgh}$ & 26.30 & $3.52 \mathrm{~b}$ & $7.74 \mathrm{c}$ & 45.52 \\
\hline Agrodhan-14 & $8.03 \mathrm{de}$ & $40.30 \mathrm{~h}-1$ & 23.40 & $2.98 \mathrm{e}$ & $6.55 \mathrm{e}$ & 45.50 \\
\hline BRRI dhan 28 & $4.23 \mathrm{ijk}$ & 31.50no & 17.50 & $0.96 \mathrm{klm}$ & $2.74 \mathrm{k}$ & 35.99 \\
\hline BRRI dhan29 & $3.53 \mathrm{jk}$ & 42.70hij & 19.60 & $1.10 \mathrm{jkl}$ & $2.75 \mathrm{k}$ & 39.97 \\
\hline BRRI dhan 47 & $3.06 \mathrm{k}$ & 44.48ghi & 19.40 & $0.88 \mathrm{mn}$ & 2.21 & 40.03 \\
\hline BRRI dhan50 & $4.53 \mathrm{hij}$ & $38.09 \mathrm{i}-\mathrm{m}$ & 18.80 & $1.15 \mathrm{jk}$ & $2.87 \mathrm{k}$ & 40.00 \\
\hline BRRI dhan 55 & $3.06 \mathrm{k}$ & $21.50 \mathrm{p}$ & 17.30 & $0.41 \mathrm{o}$ & $1.02 \mathrm{n}$ & 39.89 \\
\hline BRRI dhan58 & $4.20 \mathrm{ijk}$ & $29.20 \mathrm{o}$ & 20.20 & $0.901 \mathrm{mn}$ & 2.251 & 39.99 \\
\hline BRRI dhan59 & $6.76 \mathrm{ef}$ & $52.80 \mathrm{cde}$ & 22.80 & $2.06 \mathrm{~g}$ & $5.15 \mathrm{~g}$ & 40.04 \\
\hline BRRI dhan67 & $6.20 \mathrm{fg}$ & $36.401 \mathrm{mn}$ & 20.30 & $1.78 \mathrm{~h}$ & $4.45 \mathrm{~h}$ & 40.02 \\
\hline Binadhan-5 & $3.40 \mathrm{jk}$ & $36.70 \mathrm{k}-\mathrm{n}$ & 23.40 & $0.70 \mathrm{n}$ & $1.75 \mathrm{~m}$ & 40.02 \\
\hline Binadhan-6 & $5.80 \mathrm{fgh}$ & 31.30no & 20.50 & $1.30 \mathrm{j}$ & $3.25 \mathrm{j}$ & 37.13 \\
\hline Binadhan-8 & $2.96 \mathrm{k}$ & $28.90 \mathrm{o}$ & 16.90 & $0.47 \mathrm{o}$ & $1.17 \mathrm{n}$ & 39.85 \\
\hline Binadhan-10 & $6.20 \mathrm{fg}$ & $41.40 \mathrm{~h}-1$ & 19.60 & $1.55 \mathrm{i}$ & $3.87 \mathrm{i}$ & 38.01 \\
\hline BRRI hybrid dhan3 & $4.26 \mathrm{ijk}$ & $33.50 \mathrm{mno}$ & 20.70 & $0.941 \mathrm{~m}$ & 2.351 & 39.97 \\
\hline Agrodhan-14 & $5.00 \mathrm{ghi}$ & 32.50no & 21.20 & $1.17 \mathrm{j}$ & $2.92 \mathrm{jk}$ & 40.01 \\
\hline $\mathrm{CV} \%$ & 12.75 & 8.02 & 10.42 & 5.88 & 4.62 & 5.17 \\
\hline Level of significance & $* *$ & $* *$ & NS & $* *$ & $* *$ & NS \\
\hline
\end{tabular}

Other details are same as Table 3. 


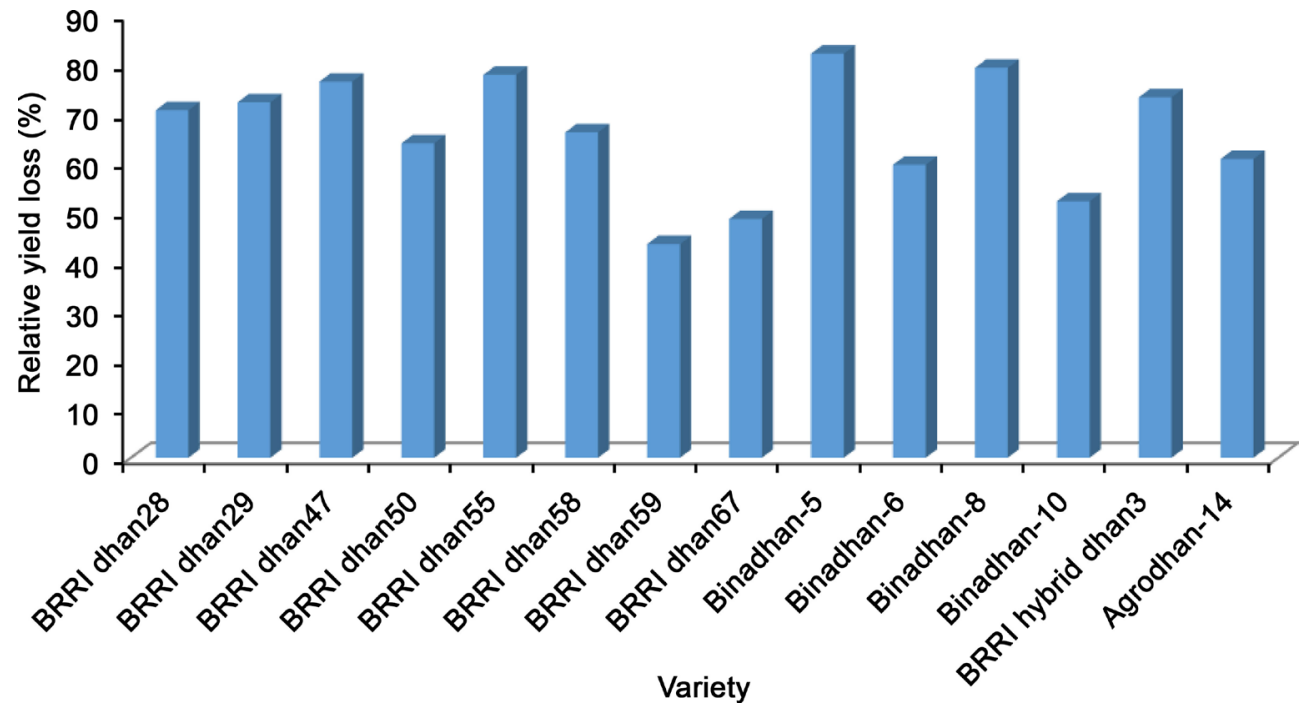

Figure 5. Effect of variety on relative yield loss (\%) of winter rice under aerobic system of cultivation.

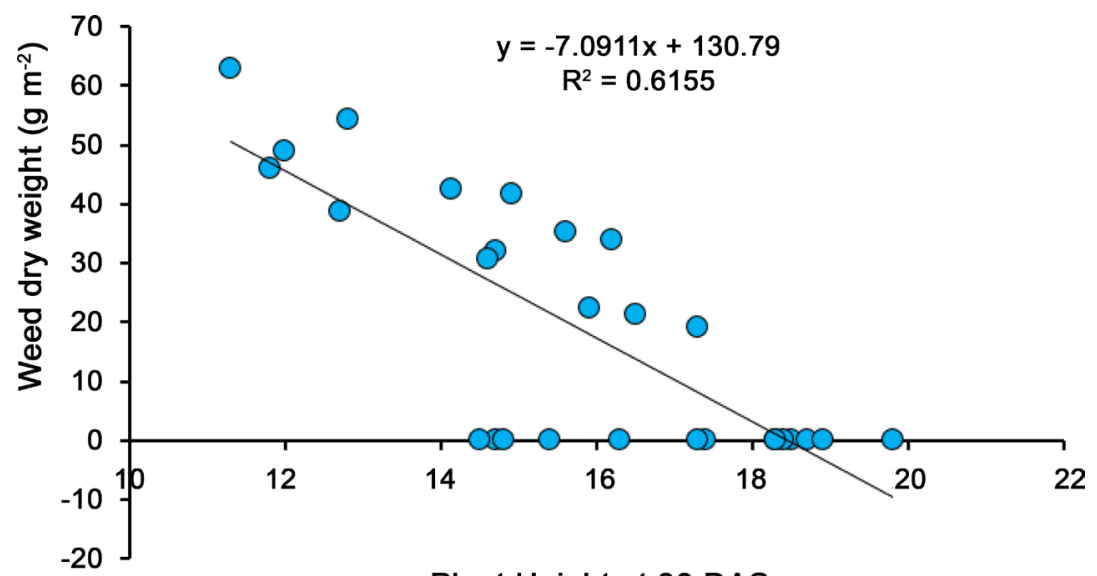

Plant Height at 30 DAS

Figure 6. Relationship between Plant height at 30 DAS and weed dry weight.

\subsection{Relationship among Traits}

Regression study showed that weed dry weight was strongly and negatively correlated with early plant height (PH30) and early visual vigor and highly (negatively) correlated with above ground crop biomass and grain yield (Figure 6). Plant height beyond 30 DAS maintained a weak negative correlation with weed dry weight (Figure 7). As expected, weed rating was strongly and positively correlated with weed dry weight, but negatively correlated with early plant height (PH30), early visual vigor and grain yield. Grain yield showed a very strong positive correlation with above ground crop biomass and highly positive correlation with early plant height $(\mathrm{PH} 30)$. While percent filled grains had maximum predictive power for grain yield $\left(\mathrm{R}^{2}=0.62\right)$ (Figure 6$)$. Weed biomass could explain grain yield by $83 \%$ (Figure 7 ) and relative yield loss by $95 \%$ (Figure 8 ). Early plant height at 30 DAS appeared to be the most important trait in predicting weed biomass $\left(\mathrm{R}^{2}=0.62\right)$ (Figure 6$)$. 


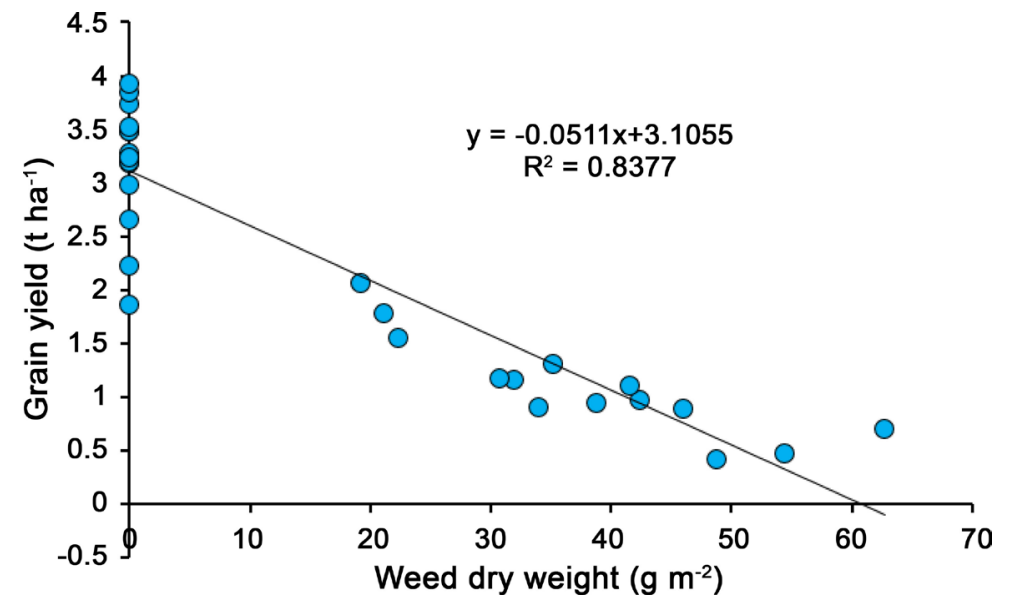

Figure 7. Relationship between weed dry weight and grain yield.

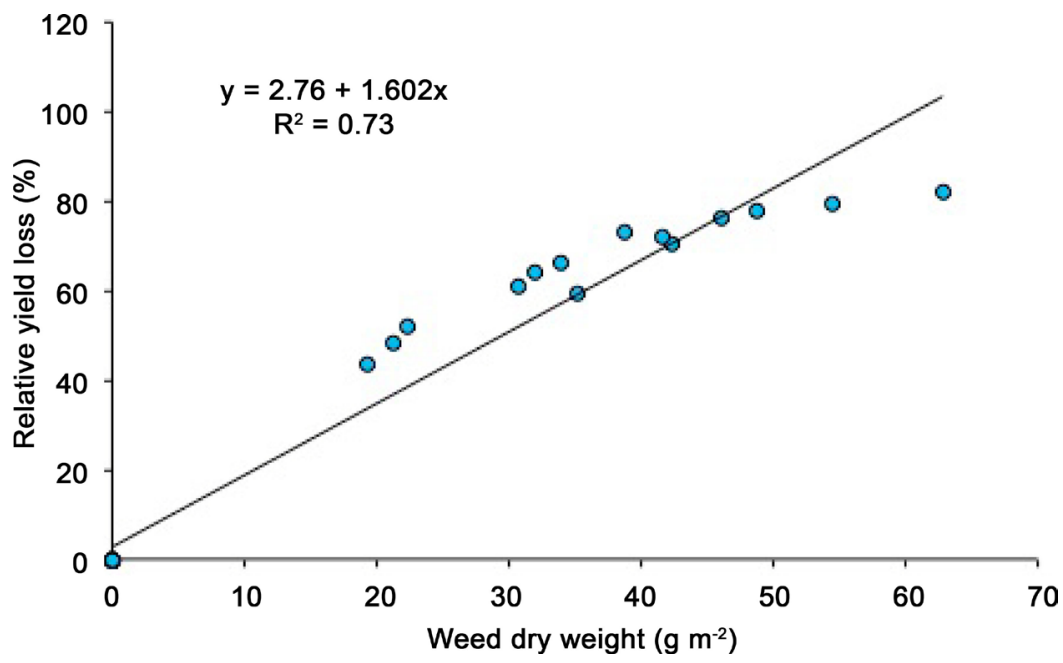

Figure 8. Relationship between weed dry weight and relative yield loss.

\section{Discussion}

The cultivars evaluated in this study varied not only in yield and weed suppressing ability, but also in all the agronomic traits measured. Although a wide variation in traits was observed among cultivars, none of them gave satisfactory yield under aerobic conditions. Binadhan-5 emerged as the most productive, whereas BRRI dhan59 appeared as the most weed suppressive rice variety. The least productive cultivar was BRRI dhan55, which was closely followed by Binadhan-8 and BRRI dhan58. Based on reports in earlier studies [9], the present study included a variety of commonly cited traits including plant height, tillering ability, early visual vigor, duration, and SPAD values. Significant variations among the varieties were recorded suggesting that selection based on those traits was practical. Plant height is considered as desirable characters for weed suppressive cultivars. Although plant height varied widely among the cultivars, early plant height i.e. height at 30DAS was strongly and negatively correlated with weed dry weight. Earlier and faster growth allowed the rice crop to compete with weeds 
for plant resources, and this was reflected in the crop yield. The results indicate that only early plant height can be considered as vital selection criteria for weed competitiveness under aerobic soil conditions. Similar type of results has also been reported by Gibson et al. [60], Caton et al. [50], Zhao et al. [61], Anwar et al. [9].

Early visual vigor or vigor index, a reliable predictor of crop biomass integrating both height and tiller number, is an important selection criterion for weed competitiveness. Yield and weed competitiveness are effectively predicted by early visual vigor. Vigor rating is rapid, non-destructive, less labor-intensive and reliable, and therefore a promising and feasible tool for making decisions on weed competitiveness [9]. In this study, early visual vigor varied widely among varieties, and its strong correlation with other parameters confirms its acceptability. It has been reported previously that early season vigor is directly linked with the competitive ability of the crop [62] [63] [64] [65] and later in the crop growing season, it confers competition against weeds [66].

The SPAD (Silicon Photon Activated Diode) meter provides a very easy, swift and non-destructive method for estimating relative leaf chlorophyll content. Higher SPAD values indicate greener healthier plants. The results showed SPAD values varied among the varieties. SPAD values were greatly reduced by weed interference and this was reflected in yield performance. Weed interference negatively and markedly affected all yield components which cumulatively impaired grain yield. The rice varieties used in the current research showed wide diversity in relative yield loss, which ranged from $43 \%$ to $82 \%$. Weed biomass was strongly and negatively correlated with grain yield, indicating that weed suppressive ability can be combined with yield potential. Anwar et al. [9] and McGregor et al. [67] also observed a similar relationship. Weed interference negatively and markedly affected all yield components which cumulatively impaired grain yield. Weed biomass was strongly and negatively correlated with grain yield, and positively correlated with relative yield loss indicating that weed suppressive ability can be combined with yield potential.

\section{Conclusion}

The findings of our study indicate that strong weed suppressive cultivars could be adopted as an integral part of sustainable weed management package aimed at reducing dependence on synthetic herbicides. However, strong weed suppressive ability does not always ensure high yields. Therefore, higher yields along with strong weed competitive cultivar should be the selection criteria for dry direct seeded aerobic rice. Competitiveness of rice cultivars against weeds will be an important key to the sustainable weed management in aerobic rice. As no cultivar in our study produced economically acceptable yield, therefore it is recommended to develop high yielding and weed competitive rice varieties that can be successfully grown following aerobic system with least weed management. 


\section{Acknowledgements}

The authors thankfully acknowledge the financial support provided by Bangladesh Agricultural University Research System (BAURES), Bangladesh Agricultural University, Mymensingh through the research project number: 2015/117/ BAU.

\section{Conflict of Interest}

The authors declare that there is no conflict of interests regarding the publication of this paper.

\section{References}

[1] Timmer, C.P. (2010) The Changing Role of Rice in Asia's Food Security. ADB Sustainable Development Working Paper Series, No. 15, Asian Development Bank, Metro Manila.

[2] FAO (Food and Agriculture Organization) (2014) FAO Statistical Databases. Food and Agriculture Organization (FAO) of the United Nations, Rome.

http://www.fao.org

[3] Jabran, K. and Chauhan, B.S. (2015) Weed Management in Aerobic Rice Systems. Crop Protection, 78, 151-163. https://doi.org/10.1016/j.cropro.2015.09.005

[4] Toriyama, K., Heong, K.L. and Hardy, B. (2005) Rice Is Life: Scientific Perspectives for the 21st Century. Proceedings of the World Rice Research Conference, Tokyo and Tsukuba, 4-7 November 2004, 590.

[5] Farooq, M., Basra, S.M.A., Ahmad, N. and Murtaza, G. (2009) Enhancing the Performance of Transplanted Course Rice by Seed Priming. Paddy and Water Environment, 7, 55-63. https://doi.org/10.1007/s10333-008-0143-9

[6] Tao, H., Brueck, H., Dittert, K., Kreye, C., Lin, S. and Sattelmacher, B. (2006) Growth and Yield Formation of Rice (Oryza sativa L.) in the Water Saving Ground Cover Rice Production System (GCRPS). Field Crops Research, 95, 1-12. https://doi.org/10.1016/j.fcr.2005.01.019

[7] Bouman, B.A.M. and Tuong, T.P. (2000) Field Water Management to Save Water and Increase Its Productivity in Irrigated Lowland Rice. Agricultural Water Management, 16, 1-20.

[8] Cantrell, R.P. and Hettel, G.P. (2005) Research Strategy for Rice in the 21st Century. Proceedings of the World Rice Research Conference, Tokyo and Tsukuba, 4-7 November 2004, 26-37.

[9] Anwar, M.P., Juraimi, A.S., Man, A., Puteh, A., Selamat, A. and Begum, M. (2010) Weed Suppressive Ability of Rice (Oryza sativa L.) Germplasm under Aerobic Soil Conditions. Australian Journal of Crop Science, 4, 706-717.

[10] Bouman, B.A.M. and Tuong, T.P. (2001) Field Water Management to Save Water and Increase Productivity in Irrigated Lowland Rice. Agricultural and Water Management, 49, 11-30. https://doi.org/10.1016/S0378-3774(00)00128-1

[11] Johnson, D.E. and Mortimer, A.M. (2005) Issues for Weed Management in Direct Seeded Rice and the Development of Decision-Support Frame-Works. In: Workshop on Direct Seeded Rice in the Rice-Wheat System of the Indo-Gangetic Plains, G B Pant University of Agriculture and Technology, Pantanagar. 
[12] Anwar, M.P., Juraimi, A.S., Puteh, A., Selamat, A., Man, A. and Hakim, M.A. (2011) Seeding Method and Rate Influence on Weed Suppression in Aerobic Rice. African Journal of Biotechnology, 10, 259-271. https://doi.org/10.5897/AJB11.060

[13] Belder, P., Bouman, B., Spiertz, J., Peng, S., Castaneda, A. and Visperas, R. (2005) Crop Performance, Nitrogen and Water Use in Flooded and Aerobic Rice. Plant and Soil, 273, 167-182. https://doi.org/10.1007/s11104-004-7401-4

[14] Li, Y. (2001) Research and Practice of Water-Saving Irrigation for Rice in China. Proceedings of the International Workshop, Wuhan, 23-25 March 2001, 135-144.

[15] Tabbal, D.F., Bouman, B.A.M., Bhuiyan, S.I., Sibayan, E.B. and Sattar, M.A. (2002) On-Farm Strategies for Reducing Water Input in Irrigated Rice: Case Studies in the Philippines. Agricultural and Water Management, 56, 93-112. https://doi.org/10.1016/S0378-3774(02)00007-0

[16] Stoop, W., Uphoff, N. and Kassam, A. (2002) A Review of Agricultural Research Issues Raised by System of Rice Intensification (SRI) from Madagascar: Opportunities for Improving Farming Systems for Resource-Poor Farmers. Agricultural Systems, 71, 249-274. https://doi.org/10.1016/S0308-521X(01)00070-1

[17] Sarkar, M.A.R., Hossain, M.Z. and Islam, A.K.M.M. (2007) Effect of Water and Fertilizer Management on the Yield and Yield Components of Boro Rice under SRI Method. Journal of Bangladesh Agricultural University, 5, 231-237.

[18] Islam, A.K.M.M., Sarkar, M.A.R. and Islam, N. (2007) Effect of Spacing and Nutrient Management in SRI Method on the Yield Components and Yield of Transplant Aman Rice. Bangladesh Journal of Crop Science, 18, 1-6.

[19] Yeasmin, S., Islam, A.K.M.M. and Sarkar, M.A.R. (2011) Integration of Fertilizer and Manure: Its Performance under the System of Rice Intensification. Lambert Academic Publisher, Saarbrücken, 1-88.

[20] Borell, A., Garside, A. and Shu, F.K. (1997) Improving Efficiency of Water for Irrigated Rice in a Semi-Arid Tropical Environment. Field Crops Research, 52, 231-248. https://doi.org/10.1016/S0378-4290(97)00033-6

[21] Juraimi, A.S., Begum, M., Yusuf, M.N.M. and Mann, A. (2010) Efficacy of Herbicides on the Control Weeds and Productivity of Direct Seeded Rice under Minimal Water Conditions. Plant Protection Quarterly, 25, 19-25.

[22] Jabran, K., Ullah, E., Hussain, M., Farooq, M., Zaman, U., Yaseen, M. and Chauhan, B.S. (2015) Mulching Improves Water Productivity, Yield and Quality of Fine Rice under Water-Saving Rice Production Systems. Journal of Agronomy and Crop Science, 201, 389-400. https://doi.org/10.1111/jac.12099

[23] Jabran, K., Ullah, E., Hussain, M., Farooq, M., Haider, N. and Chauhan, B.S. (2015) Water Saving, Water Productivity and Yield Outputs of Fine-Grain Rice Cultivars under Conventional and Water-Saving Rice Production Systems. Experimental Agriculture, 51, 567-581. https://doi.org/10.1017/S0014479714000477

[24] Bastiaans, L., Kropff, M.J., Goudriaan, J. and Laar, H.H. (1997) Design of Weed Management Systems with a Reduced Reliance on Herbicides Poses New Challenges and Prerequisites for Modeling Crop-Weed Interactions. Field Crops Research, 67, 161-179. https://doi.org/10.1016/S0378-4290(00)00091-5

[25] Oerke, E.C. and Dehne, H.W. (2004) Safe Guarding Production Losses in Major Crops and the Role of Crop Protection. Crop Protection, 23, 275-285. https://doi.org/10.1016/j.cropro.2003.10.001

[26] Karim, S.M.R., Man, A.B. and Sahid, I.B. (2004) Weed Problems and Their Management in Rice Fields of Malaysia: An Overview. Weed Biology Management, 4, 177-186. https://doi.org/10.1111/j.1445-6664.2004.00136.x 
[27] Mahajan, G. and Chauhan, B.S. (2011) Effects of Planting Pattern and Cultivar on Weed and Crop Growth in Aerobic Rice System. Weed Technology, 25, 521-525. https://doi.org/10.1614/WT-D-11-00025.1

[28] Rao, A.N., Johnson, D.E., Sivaprasad, B., Ladha, J.K. and Mortimer, A.M. (2007) Weed Management in Direct Seeded Rice. Advances in Agronomy, 93, 153-255. https://doi.org/10.1016/S0065-2113(06)93004-1

[29] Azmi, M. (1992) Competitive Ability of Barnyard Grass in Direct Seeded Rice. Teknologi Padi, 8, 19-25.

[30] Balasubramanian, V. and Hill, J.E. (2002) Direct Seeding of Rice in Asia: Emerging Issues and Strategic Research Needs for the 21st Century. In: Pandey, et al., Eds., Direct Seeding. Research Strategies and Opportunities, IRRI, Los Banos, 15-39.

[31] Jayadeva, H.M., Bhairappanavar, S.T., Hugar, A.Y., Rangaswamy, B.R., Mallikarjun, G.B., Malleshappa, C. and Naik, D.C. (2011) Integrated Weed Management in Aerobic Rice (Oryza sativa L.). Agricultural Science Digest, 31, 58-61.

[32] Swanton, C.J. and Weise, S.F. (1991) Integrated Weed Management: The Rationale and Approach. Weed Technology, 5, 657-663.

[33] O’Donovan, J.T., Harker, K.N., Clayton, G.W., Newman, J.C., Robinson, D. and Hall, L.M. (2001) Barley Seeding Rate Influence the Effects of Variable Herbicide Rates. Weed Science, 49, 746-754. https://doi.org/10.1614/0043-1745(2001)049[0746:BSRITE]2.0.CO;2

[34] Grichar, W.J., Bessler, B.A. and Brewer, K.D. (2004) Effect of Row Spacing and Herbicide Dose on Weed Control and Grain Sorghum Yield. Crop Protection, 23, 263-267. https://doi.org/10.1016/j.cropro.2003.08.004

[35] Dass, A., Shekhawat, K., Choudhary, A.K., Sepat, S., Rathore, S.S., Mahajan, G. and Chauhan, B.S. (2017) Weed Management in Rice using Crop Competition-A Review. Crop Protection, 95, 45-52. https://doi.org/10.1016/j.cropro.2016.08.005

[36] Van der Meulen, A. and Chauhan, B.S. (2017) A Review of Weed Management in Wheat using Crop Competition. Crop Protection, 95, 38-44. https://doi.org/10.1016/j.cropro.2016.08.004

[37] Heap, I. (2017) The International Survey of Herbicide Resistant Weeds. http://www.weedscience.com

[38] Aktar, M.W., Sengupta, D. and Chowdhury, A. (2009) Impact of Pesticides Use in Agriculture: Their Benefits and Hazards. Interdisciplinary Toxicology, 2, 1-12. https://doi.org/10.2478/v10102-009-0001-7

[39] Fischer, A.J., Ateh, C.M., Bayer, D.E. and Hill, J.E. (2000) Herbicide-Resistant (Echinochloa oryzoides) and (E. phyllopogon) in California Oryza sativa Fields. Weed Science, 48, 225-230. https://doi.org/10.1614/0043-1745(2000)048[0225:HREOAE]2.0.CO;2

[40] Pell, M., Stenberg, B. and Torstensson, L. (1998) Potential Denitrification and Nitrification Tests for Evaluation of Pesticide Effects in Soil. Ambio, 27, 24-28.

[41] Rahman, M.M., Sahid, I.B. and Juraimi, A.S. (2010) Study of Resistant Biotypes of Echinochloa crussgalli in Malaysia. Australian Journal of Crop Science, 4, 107-115.

[42] Roder, W. (2001) Slash-and-Burn Rice Systems in the Hills of Northern Lao PDR. In: Description, Challenges, and Opportunities, IRRI, Los Banos, 201.

[43] Jannink, J.L., Orf, J.H., Jordan, N.R. and Shaw, R.G. (2000) Index Selection for Weed Suppressive Ability in Soybean. Crop Science, 40, 1087-1094. https://doi.org/10.2135/cropsci2000.4041087x 
[44] Dingkuhn, M., Johnson, D.E., Sow, A. and Audebert, A.Y. (1999) Relationships between Upland Rice Canopy Characteristics and Weed Competitiveness. Field Crops Research, 61, 79-95. https://doi.org/10.1016/S0378-4290(98)00152-X

[45] Fischer, A.J., Ramirez, H.V., Gibson, K.D. and Pinheiro, B.D.S. (2001) Competitiveness of Semi-Dwarf Upland Rice Cultivars against Palisade Grass (Brachiaria brizantha) and Signal Grass (B. decunmbens). Agronomy Journal, 93, 967-973. https://doi.org/10.2134/agronj2001.935967x

[46] Gibson, K.D. and Fischer, A.J. (2004) Competitiveness of Rice Cultivars as a Tool for Crop-Based Weed Management. In: Inderjit, Ed., Weed Biology and Management, Kulwer Academic Publishers, Dordrecht, 517-532. https://doi.org/10.1007/978-94-017-0552-3_25

[47] Mahajan, G., Brar, L.S. and Sardana, V. (2004) Efficacy of Clodinafop against Isoproturon Resistant Phalaris Minor in Relation to Wheat Cultivars and Spacing. Indian Journal of Weed Science, 36, 166-170.

[48] Zhao, D.L., Atlin, G.N., Bastiaans, L. and Spiertz, J.H.J. (2006) Comparing Rice Germplasm Groups for Growth, Grain Yield and Weed Suppressive Ability under Aerobic Soil Conditions. Weed Research, 46, 444-452. https://doi.org/10.1111/j.1365-3180.2006.00529.x

[49] Juraimi, A.S., Uddin, M.K., Anwar, M.P., Mohamed, M.T.M., Ismail, M.R. and Man, A. (2013) Sustainable Weed Management in Direct Seeded Rice Culture: A Review. Australian Journal of Crop Science, 7, 989-1002.

[50] Caton, B.P., Cope, A.E. and Mortimer, M. (2003) Growth Traits of Diverse Rice Cultivars under Severe Competition: Implications for Screening for Competitiveness. Field Crops Research, 83, 157-172. https://doi.org/10.1016/S0378-4290(03)00072-8

[51] Anwar, M.P., Juraimi, A.S., Samedani, B., Mohamed, M.T.M., Uddin, M.K., Hasan, A.K. and Hossain, M.D. (2014) Integrating Cultural and Chemical Control Methods for Higher Weed Control Efficiency and Better Performance of Aerobic Rice. Research on Crops, 15, 1-13. https://doi.org/10.5958/j.2348-7542.15.1.001

[52] Chauhan, B.S. (2012) Weed Ecology and Weed Management Strategies for Dry-Seeded Rice in Asia. Weed Technology, 26, 1-13.

https://doi.org/10.1614/WT-D-11-00105.1

[53] Anwar, M.P., Juraimi, A.S., Mohamed, M.T.M., Uddin, M.K., Samedani, B., Puthe, A. and Man, A. (2013) Integration of Agronomic Practices with Herbicides for Sustainable Weed Management in Aerobic Rice. The Scientific World Journal, 2013, 1-12. https://doi.org/10.1155/2013/916408

[54] FAO-UNDP (Food and Agriculture Organization-United Nations Development Programme) (1988) Land Resources Appraisal of Bangladesh for Agricultural Development. Agro-Ecological Regions of Bangladesh, BGD/81/035. Technical Report No. 2, FAO, Rome.

[55] Islam, A.K.M.M., Meirvenne, M.V. and Islam, M.M. (2011) Proximal Soil Sensing System for Paddy Field Variability Mapping: The Potential of a Proximal Soil Sensor for Soil Properties Mapping. VDM Verlag Dr. Muller, Saarbrucken, 1-60.

[56] BRRI (Bangladesh Rice Research Institute) (2017) Adhunik Dhaner Chash. 18th Edition, 8-10.

[57] BINA (Bangladesh Institute of Nuclear Agriculture) (2012) BINA Profile. 4th Edition, 24-26. 
[58] Janiya, J.D. and Moody, K. (1989) Weed Populations in Transplanted Wet-Seeded Rice as Affected by Weed Control Method. Tropical Pest Management, 35, 8-11. https://doi.org/10.1080/09670878909371311

[59] Gomez, K.A. and Gomez, A.A. (1984) Statistical Procedures for Agricultural Research. John Wiley and Sons, New York, Chichesten, Brisbane, Toronto, 97-215.

[60] Gibson, K.D., Hill, J.E., Foin, T.C., Caton, B.P. and Fischer, A.J. (2001) Water Seeded Rice Cultivars Differ in Ability to Interfere with Water Grass. Agronomy Journal, 93, 326-332. https://doi.org/10.2134/agronj2001.932326x

[61] Zhao, D.L., Atlin, N., Bastiaans, L. and Spiertz, J.H.J. (2006) Developing Selection Protocols for Weed Competitiveness in Aerobic Rice. Field Crops Research, 97, 272-285. https://doi.org/10.1016/j.fcr.2005.10.008

[62] Bertholdsson, N.O. (2005) Early Vigour and Allelopathy-Two Useful Traits for Enhanced Barley and Wheat Competitiveness against Weeds. Weed Research, 45, 94-102. https://doi.org/10.1111/j.1365-3180.2004.00442.x

[63] Saito, K., Azoma, K. and Rodenburg, J. (2010) Plant Characteristics Associated with Weed Competitiveness of Rice under Upland and Lowland Conditions in West Africa. Field Crops Research, 116, 308-317. https://doi.org/10.1016/j.fcr.2010.01.008

[64] Mahajan, G., Ramesha, M.S. and Chauhan, B.S. (2014) Response of Rice Genotypes to Weed Competition in Dry Direct-Seeded Rice in India. The Scientific World Journal, 2014, 1-8. https://doi.org/10.1155/2014/641589

[65] Zhao, D.L., Atlin, G.N., Bastiaans, L. and Spiertz, J.H.J. (2006) Cultivar Weeds Competitiveness in Aerobic Rice: Heritability, Correlated Traits, and the Potential for Indirect Selection in Weed-Free Environments. Crop Science, 46, 372-380. https://doi.org/10.2135/cropsci2005.0192

[66] Huel, D.G. and Hucl, P. (1996) Genotypic Variation for Competitive Ability in Spring Wheat. Plant Breeding, 115, 325-329. https://doi.org/10.1111/j.1439-0523.1996.tb00927.x

[67] McGregor, J.T., Roy, J.R., Smith, J.R. and Talbert, R.E. (1988) Broadleaf Signal Grass (Brachiaria platyphylla) Duration of Interference in Rice (Oryza sativa). Weed Science, 36, 747-750. 